\title{
Detecting In-Vitro Colistin Resistance- A Comparative Study Between Broth Microdilution Versus Vitek-2 For Colistin Susceptibility Testing
}

\author{
Hena Butta ${ }^{1 *}$, Leena Mendiratta ${ }^{1}$, Raman Sardana ${ }^{1}$, Kirti Gilotra ${ }^{1}$, Sana Hasan ${ }^{2}$ \\ Sudha Kansal ${ }^{3}$ and Suranjit Chatterjee ${ }^{4}$ \\ ${ }^{1}$ Department of Microbiology, Indraprastha Apollo Hospitals, New Delhi, India \\ ${ }^{2}$ Dept of Microbiology, School of Biotechnology, IFTM University, Moradabad, India \\ ${ }^{3}$ Department of Critical Care, Respiratory and Sleep Medicine Indraprastha Apollo Hospitals, New Delhi, India \\ ${ }^{4}$ Department of Internal Medicine, Indraprastha Apollo Hospitals, New Delhi, India
}

\begin{abstract}
Background: Susceptibility testing for polymyxins is a great challenge for a Clinical Microbiology laboratory. There are several methodological issues associated with MIC (Minimum Inhibitory Concentration) determination of colistin.

Methods: In our study, we have compared the results of colistin susceptibility testing by Automated system (Vitek-2, Biomerieux, France) with the reference Broth Microdilution method (BMD) to identify the type of discrepancies by Vitek-2 method and thus develop a practical and accurate approach for colistin susceptibility testing in a Clinical Microbiology laboratory. A total of 730 strains of Gram negative bacteria [Escherichia coli (325), Klebsiella sp.(346), Acinetobacter baumanii complex (37) and Pseudomonas aeruginosa (22)] from 485 patents were tested simultaneously by BMD and Vitek-2 method for colistin susceptibility testing.

Results: The Essential agreement (EA), Categorical agreement (CA), Very major error (VME) and Major error (ME) rates for Klebsiella sp.were $87.3 \%, 89.3 \%, 8 \%$ and $2.3 \%$ respectively, for Escherichia coli were $88.3 \%, 89.5 \%, 9.2 \%$ and $1.2 \%$ respectively, for Acinetobacter baumannii complex were $89.1 \%, 91.8 \%, 8.1 \%$ and $0 \%$ respectively, for Pseudomonas aeruginosa were 68.1\%, 72.7\%, 0\% and 27.2\% respectively.

Conclusions: Colistin susceptibility testing by Vitek-2 method is an easily adoptable method and the results of Vitek-2 with reference to BMD are acceptable to a great extent in Klebsiella sp., Escherichia coli and Acinetobacter baumanii complex. So, we believe that Vitek-2 method may be used for colistin susceptibility testing in low risk patients. However, BMD should be used in high risk immunosupressed and immunocompromised patients who are admitted in critical care units. For Pseudomonas aeruginosa, BMD should be routinely used.
\end{abstract}

Keywords: Colistin, Susceptibility, Broth Microdilution, Vitek-2

\section{Introduction}

Polymyxins are a group of polypeptide antibiotics that were first isolated in 1947 from a spore-bearing soil bacillus (Bacillus polymyxa). There are several chemically different polymyxins which have been isolated from different strains of this bacillus and these are Polymyxin A to Polymyxin E. But, only polymyxin B and polymyxin E (colistin) have been used clinically. Polymyxin B and colistin differs from each other only by a single aminoacid in the peptide ring. The antibacterial spectrum of Polymyxins is mainly against Gram negative organisms viz Escherichia coli, Klebsiella sp., Enterobacter sp., Citrobacter sp., Acinetobacter sp. and Pseudomonas aeruginosa. Amongst Enterobacteriacae, the members of Tribe Proteae (Proteus, Providencia, Morganella) and Serratia sp. are inherently resistant to Polymyxins. ${ }^{[1]}$

With the increase in multidrug resistance amongst Gram negative bacilli, Colistin has become the last resort for the treatment of infections caused by these microorganisms, particularly carbapenem-resistant Gram negative bacteria. Colistin is administered as an inactive prodrug, colistin methanesulfonate (colistimethate). In aqueous media and biological fluids, this prodrug is converted into colistin and several inactive methanesulfonated compounds.

Susceptibility testing for polymyxins is a great challenge for a clinical laboratory because of challenges in testing of polymyxins which include less diffusion of polymyxins into agar, inherent cationic properties of polymyxins, the occurrence of heteroresistance to polymyxins in many species, and lack of a reliable reference method that may allow reliable comparisons of commercial tests..$^{[2]}$ Because of several methodological issues associated with MIC testing of colistin, CLSI-EUCAST joint Polymyxin Breakpoints Working Group recommends use of Broth microdilution (BMD) method for susceptibility testing of colistin ${ }^{[3]}$ However, BMD method has not been adaptable 
for a clinical Microbiology laboratory because it is manual and quite labor intensive.

The other methods for susceptibility testing of polymyxins include Disk diffusion method, E-test method, and automated methods (Vitek-2, Phoenix, Microscan etc). Although both Disk diffusion method and E-test method are less labor intensive and easy to perform but, both of them are associated with false susceptibility results when compared with BMD method. ${ }^{[4,5]}$ Vitek-2 method has also been found to have low sensitivity in detecting colistin resistance in Gram negative organisms. ${ }^{[5,6]}$ The studies pertaining to evaluation of other automated systems like Phoenix, Microscan and Sensititre systems with respect to BMD method for colistin susceptibility are scarce. ${ }^{[2]}$

We at our tertiary health care set-up are using Vitek-2 for antimicrobial susceptibility testing. So, keeping the pros and cons of automated systems especially with respect to colistin susceptibility testing in mind, we planned to compare the results of colistin susceptibility testing by Vitek-2 method with the reference Broth microdilution method to establish a practical and accurate approach for colistin susceptibility testing in a Clinical Microbiology laboratory.

\section{Material and Methods}

This study was done on 730 clinically significant strains of Gram-negative bacteria isolated from clinical specimens of the patients in a tertiary heath care set-up between August 2018 to December 2018. The clinical specimens included Blood, Pus/Tissue, Body fluids, Respiratory specimens and Urine. These isolates were from derived from both in-patients and out-patients. These patients comprised of mixed population of immunocompetent, immunosupressed/immunocompromised, critically ill patients. The Gram negative bacteria included Escherichia coli (325), Klebsiella sp. (346), Acinetobacter baumanii complex (37) and Pseudomonas aeruginosa (22). These 730 strains were the non-repeat isolates from the same sample of 485 patients. The determination of MIC value of colistin by Vitek-2 method was done as per the manufacturer instructions.

A. BMD procedure was done as per CLSI guidelines. ${ }^{[7,8]}$ Stock solution of colistin in concentration of $5120 \mu \mathrm{g} /$ $\mathrm{ml}$ was prepared by dissolving $102.4 \mathrm{mg}$ of colistin sulfate powder (Sigma-Aldrich; Potency $=500 \mu \mathrm{g}$ / $\mathrm{mg}$ ) in $10 \mathrm{ml}$ of sterile water. Colistin stock solution was filter sterilized and aliquoted in smaller volumes and stored at $-60{ }^{\circ} \mathrm{C}$. Cation adjusted Mueller Hinton Broth (CAMHB) was prepared as per the manufacturer instructions. BBL ${ }^{\mathrm{TM}}$ Mueller Hinton II Broth Cation Adjusted (Becton, Dickinson and
Company, USA). Fresh dilutions $(256 \mu \mathrm{g} / \mathrm{ml}$ to 0.5 $\mu \mathrm{g} / \mathrm{ml}$ ) of colistin was made with every batch of test and Broth Microdilution plates were prepared with different concentrations of colistin solution. Bacterial suspension was prepared in the concentration of $5 \mathrm{x}$ $10^{5} \mathrm{CFU} / \mathrm{ml}$ and inoculated in microbroth plate. The last two wells of each row of microbroth plate acted as Growth control and Sterility control. Growth control well contained only adjusted bacterial suspension and Sterility control well contained only Cation adjusted Mueller Hinton Broth (CAMHB) which was used to prepare various dilutions. In every batch Quality control strains are used as control. The controls used were Escherichia coli ATCC35218, Escherichia coli ATCC25922 and Pseudomonas aeruginosa ATCC27853.The microbroth plate was then incubated at $37^{\circ} \mathrm{C}$ for $16-18 \mathrm{hrs}$. Reading of the test strains was taken only after satisfactory reading of the control strains. The minimum concentration of colistin which inhibits the visible growth of the bacteria was taken as its MIC. Interpretation of the results was done as per CLSI guidelines 2018. Based on epidemiological cut-off value for Enterobacteriaceae, Klebsiella sp. and Escherichia coli were considered as Sensitive if $\mathrm{MIC}$ value was $<=2 \mu \mathrm{g} / \mathrm{ml}$ and as Resistant if MIC value was $>=4 \mu \mathrm{g} / \mathrm{ml}$. For Pseudomonas aeruginosa and Acinetobacter sp. MIC value of $<=2 \mu \mathrm{g} / \mathrm{ml}$ was interpreted as Sensitive and MIC value of $>=4 \mu \mathrm{g} / \mathrm{ml}$ was interpreted as Resistant. ${ }^{[7]}$

B. Comparison between Vitek-2 and BMD results: BMD was considered as gold standard and the MIC values obtained by Vitek-2 was compared with BMD Essential Agreement (EA), Categorical Agreement (CA), Very Major Error rate (VME) and Major Error rates (ME) were calculated. If the MIC of the isolates by Vitek-2 were within +/- one doubling dilution in comparison to BMD, then the two methods were considered to be in essential agreement for that isolate. Those isolates which fall in the same category of interpretation were considered to be in Categorical agreement. If the isolate was resistant by BMD and susceptible by Vitek-2, it was considered as very major error. If the isolate was susceptible by BMD but resistant by Vitek-2 it was considered as Major error. $\mathrm{EA}, \mathrm{CA}, \mathrm{VME}$ rate and $\mathrm{ME}$ rate were calculated as percentage. ${ }^{[9]}$

Acceptable performance between the two methods was evaluated according to criteria established by the International Organization for Standardization: $>90 \%$ for essential or category agreement and $<3 \%$ for $\mathrm{VME}$ or ME. ${ }^{[10]}$ 
C. Re-confirmation of discrepant results: Re-confirmation of the discrepant results between Vitek-2 and BMD method (Both VME and ME) was done by repeat testing.

\section{Results}

A total of 730 Gram negative bacteria isolated from various clinical specimens were studied for colistin susceptibility by Broth microdilution method and Vitek-2 method.

Out of 730 Gram negative bacteria, 346 strains were Klebsiella pneumoniae, 325 strains were Escherichia coli, 22 strains were Pseudomonas aeruginosa and 37 strains of Acintobacter baumannii complex.

Colistin Resistance: The overall resistance to Colistin amongst Gram negative bacilli was found to be $19.17 \%$ (140/730) by gold standard BMD method. For Enterobacteriaceae (Escherichia coli and Klebsiella sp.), the resistance to colistin was found to be $19.5 \%$ $(131 / 671)$ and for Non fermenters (Acinetobacter sp. and Pseudomonas aeruginosa), the resistance to colistin was found to be $15.2 \%(09 / 59)$.

The detailed results of these isolates are presented below:

Escherichia coli The total number of Escherichia coli strains which were sensitive to colistin by Vitek-2 method was 315 and by BMD were 288. Out of 10 strains which were found to be resistant by Vitek-2 method, six strains were resistant by BMD method also, but four of these resistant strains were found to be sensitive by BMD. Out of 35 strains detected resistant by MBD, six were also detected resistant by Vitek-2 method. Vitek-2 method failed to detect resistance in 29 strains of Escherichia coli.

Klebsiella sp.: The total number of Klebsiella sp. strains which were sensitive to colistin by Vitek-2 method was 273 and by BMD were 252. Out of 73 strains which were found to be resistant by Vitek-2 method, 66 were found to be resistant by BMD method also i.e. seven strains which were detected as resistant by Vitek-2 method were found to be sensitive by MBD method. Out of 94 strains detected as resistant by BMD method, 66 were also detected by
Vitek-2 method i.e. Vitek-2 failed to detect resistance in 28 strains of Klebsiella sp..

\section{Acinetobacter sp.}

The total number of Acinetobacter strains which were found to be sensitive to colistin by Vitek-2 method was 34 and by BMD was 31 . The three strains detected as resistant by Vitek- 2 method were also detected as resistant by BMD method. In comparison to BMD method, Vitek-2 method failed to detect colistin resistance in three cases.

\section{Pseudomonas aeruginosa}

The total number of Pseudomonas aeruginosa strains which were found to be sensitive to colistin by Vitek-2 method was 13 and by BMD method were 19. However, six strains detected resistant by Vitek-2 method were found to be sensitive by BMD method.

The performance of Vitek-2 method with respect to BMD method is shown in table-1.

\section{Discussion}

Colistin is the mainstay of treatment in patients with Carbapenem resistant Gram negative bacteria infections. However, there are controversies in susceptibility test results using different methods. In the present study, a comparison between automated and user friendly Vitek- 2 method and gold standard BMD method for colistin have been done in a clinical microbiology laboratory. A total of 730 Gram negative bacteria routinely isolated from various clinical specimens were tested for colistin susceptibility by Microbroth dilution method and Vitek-2 methods. A discrepancy between two test methods was found to be $8.0 \%, 9.5 \%, 8.1 \%, 27.27 \%$ for Klebsiella sp., Escherichia coli, Acinetobacter sp. and Pseudomonas aeruginosa respectively. The discrepancy for Pseudomonas aeruginosa is apparently more because of the lower number of the isolates considered in the study. But, the important thing to note for Pseudomonas aeruginosa is that MBD detected Pseudomonas aeruginosa strains as colistin sensitive, however, these strains were detected as colistin resistant by Vitek-2 method. This is in contrast to Klebsiella sp., Escherichia coli and Acinetobacter sp. which were more detected as colistin resistant by BMD method than Vitek-2 method.

Table 1: Comparative between Vitek-2 and BMD method results for Colistin susceptibility testing in major Gram negative bacteria isolated from clinical specimens.

\begin{tabular}{|l|l|l|l|l|l|}
\hline Gram negative bacteria & Number of strains tested & EA & CA & VME & ME \\
\hline Klebsiella sp. & 346 & $87.3 \%$ & $89.3 \%$ & $8 \%$ & $2.3 \%$ \\
\hline Escherichia coli & 325 & $88.3 \%$ & $89.5 \%$ & $9.2 \%$ & $1.2 \%$ \\
\hline Acintobacter baumannii complex & 37 & $89.1 \%$ & $91.8 \%$ & $8.1 \%$ & $0 \%$ \\
\hline Pseudomonas aeruginosa & 22 & $68.1 \%$ & $72.7 \%$ & $0 \%$ & $27.2 \%$ \\
\hline
\end{tabular}


In our study, the categorical agreement of Vitek-2 for Acinetobacter baumanii complex was acceptable and that of Escherchia coli and Klebsiella pneumoniae was marginally acceptable. However, Vitek-2 was not in CA agreement with BMD in case of Pseudomonas aeruginosa. The Categorical and Essential disagreement in Pseudomonas aeruginosa was because of major errors and not due to very major errors. This disagreement in Pseudomonas aeruginosa may be because of the lesser number of Pseudomonas aeruginosa strains considered in our study. In our study, for Acinetobacter baumannii complex, EA/CA was found to be $89.1 \% / 91.8 \%$ and VME $/ \mathrm{ME}$ rates were $8.1 \% / 0 \%$. Vourli S et al in their study found the EA/CA between Vitek-2 and BMD to be $88.9 \% / 89.7 \%$ in carbapenem resistant Acinetobacter baumanii clinical isolates. ${ }^{[11]}$ In a study by Bakthavatchalam YD et al, the EA, CA, VME rate and ME rate was found to be $69 \%$, $93 \%, 8 \%$ and $0 \%$ respectively for Klebsiella pneumoniae and $81 \%, 97 \%, 3 \%$ and $0 \%$ respectively for Acinetobacter baumannii. $^{[12]}$ Dafopoulou $\mathrm{K}$ et al found $75.6 \%$ EA and $100 \%$ CA with nil ME and VME rates in Klebsiella pneumoniae isolates. The EA, CA, VME, ME rates were found to be $85 \%, 90 \%, 0 \%$ and $10 \%$ respectively in Acinetobacter baumannii. The isolates in their study were mainly colistin resistant. ${ }^{[13]}$

Colistin gradient diffusion tests (E-tests and MIC strip tests) have also not been found to be suitable for the measurement of colistin MIC in clinical isolates in various studies. ${ }^{[12,13,14]}$

It is also important to test all the MDR isolates by BMD method of Colistin susceptibility testing because false sensitive or false resistant would put the patient on inappropriate antibiotics. We have considered both carbapenem susceptible (non MDR) and nonsusceptible (MDR) bacterial strains in our study and colistin susceptibility testing by BMD was performed simultaneously with Vitek-2 method on the same day of isolation as a routine susceptibility testing method for colistin. This is in contrast to other studies where the authors have done the susceptibility testing on carbapenem non susceptible (MDR) stocked strains which were revived at the time of testing whereby changes can occur due to subcultures and further population diversity. ${ }^{[13]}$

\section{Conclusions}

In our study, colistin susceptibility testing was done simultaneously with Vitek-2 and BMD method and the agreement between Vitek-2 results and BMD was marginally acceptable for Escherichia coli and Klebsiella pneumoniae and acceptable in Acinetobacter baumannii complex. In a tertiary health care facility, colistin is used both empirically and therapeutically because of the type of patient population who are generally referred cases from primary/secondary health care facilities and already on highend antibiotics or immunosupressed or post-transplant or malignancy patients who are on antimicrobial prophylaxis or treatment. In these patients there are chances of isolation of multidrug resistant bacteria where colistin is the only drug of choice therapeutically. In immunosuppressed or immunocompromised patients, colistin susceptibility testing should be carried out and interpreted routinely using gold standard microbroth dilution method for deciding the optimum choice of drug for all the indicated organisms except Pseudomonas aeruginosa which requires further large scale testing. Based on the acceptable agreement between Vitek-2 and BMD method in our study, colistin susceptibility testing done by Vitek- 2 method in low risk patients is acceptable.

\section{References}

1. Falagas ME, Kasiakou SK. Colistin: the revival of polymyxins for the management of multidrug-resistant Gram-negative bacterial infections. Clin Infect Dis 2005;40:1333-1341.

2. Poirel L, Jayol A, Nordmann P. Polymyxins: antibacterial activity, susceptibility testing, and resistance mechanisms encoded by plasmids or chromosomes. Clin Microbiol Rev 2017;30:557-596.

3. http://www.eucast.org/fileadmin/src/media /PDFs/EUCAST _files/General_documents/ Recommendations_for_MIC determination_of_colistin_March_2016.pdf).

4. Hindler JA, Humphries RM. 2013. Colistin MIC variability by method for contemporary clinical isolates of multidrugresistant Gram-negative bacilli. J Clin Microbiol 51:1678 -1684 .

5. Lo-Ten-Foe JR, de Smet AM, Diederen BM, Kluytmans JA, van Keulen PH. Comparative evaluation of the VITEK 2, disk diffusion, $\mathrm{E}$ test, broth microdilution, and agar dilution susceptibility testing methods for colistin in clinical isolates, including heteroresistant Enterobacter cloacae and Acinetobacter baumannii strains. Antimicrob Agents Chemother 2007;51:3726-3730.

6. Tan TY, Ng SY. Comparison of Etest, Vitek and agar dilution for susceptibility testing of colistin. Clin Microbiol Infect 2007;13:541-544.

7. CLSI. Performance Standards for Antimicrobial Susceptibility Testing. 28th ed. CLSI supplement M100. Wayne,PA: Clinical and Laboratory Standards Institute; 2018.

8. CLSI. Methods for Dilution Antimicrobial Susceptibility Tests for Bacteria that grow aerobically. 11th ed. CLSI standard M07. Wayne,PA: Clinical and Laboratory Standards Institute;2018. 
9. Patel JB, Sharp S, Novak-Weekley S. Verification of Antimicrobial Susceptibility methods: a Practical approach. Clinical Microbiology Newsletter 2013;35(13):103-9.

10. International Organization for Standardization. 2007. ISO 20776-2: 2007(E). Clinical laboratory testing and in vitro diagnostic test systems. Susceptibility testing of infectious agents and evaluation of performance of antimicrobial susceptibility test devices. Part 2: evaluation of performance of antimicrobial susceptibility test devices. International Organization for Standardization, Geneva, Switzerland.

11. Vourli S, Dafopoulou K, Vrioni G, Tsakris A, Pournaras S. Evaluation of two automated systems for colistin susceptibility testing of carbapenem-resistant Acinetobacter baumannii clinical isolates.J Antimicrob Chemother. 2017;72(9):2528-2530

12. Bakthavatchalam YD, Veeraraghavan B, Shankar A, Thukaram B, Krishnan DN. Evaluation of colistin and polymyxin B susceptibility testing methods in Klebsiella pneumoniae and Acinetobacter baumannii. J Infect Dev Ctries 2018;12(6):504-507

13. Dafopoulou K, Zarkotou O, Dimitroulia E, Hadjichristodoulou C, Gennimata V, Pournaras S, Tsakrisa A. Comparative Evaluation of Colistin Susceptibility Testing Methods among Carbapenem-Nonsusceptible Klebsiella pneumoniae and Acinetobacter baumannii clinical isolates. Antimicrobial agents and Chemotherapy 2015;59:46254630.

14. Matuschek E, Åhman J, Webster C, Kahlmeter G. Antimicrobial susceptibility testing of colistin - evaluation of seven commercial MIC products against standard broth microdilution for Escherichia coli, Klebsiella pneumoniae, Pseudomonas aeruginosa, and Acinetobacter spp. Clinical Microbiology and Infection 2018;24:865-870.

*Corresponding author:

Dr. Hena Butta, Sr. Consultant, Department of Microbiology, Indraprastha Apollo Hospitals, New Delhi, INDIA

Phone: +91 8447233605

Email: henavasdeva@yahoo.com 